ranged from 11\%-61\%; no guideline was deemed suitable for use. Only Swiss and Canadian guidelines were considered useable with significant modifications.

Conclusion Several international guidelines on perinatal care of 22-25 wk GA infants exist. Using the AGREE-II tool, we identified many deficits in the quality of these guidelines. Not a single guideline was deemed suitable for use using the AGREE-II tool. Use of poorly developed guidelines may be detrimental to decision-making, thus there is a need for transparent and rigorous guidelines regarding the perinatal care of 22-25 wk GA infants.

\section{PS-050 QUALITY OF LIFE FOR PARENTS OF VERY-LOW BIRTH WEIGHT INFANTS ENROLLED IN A CLINICAL STUDY}

${ }^{1} \mathrm{~T}$ Nordheim, ${ }^{2} \mathrm{~T}$ Rustøen, ${ }^{1} \mathrm{~B}$ Nakstad. 'Department of Children and Adolescents Medicine, Akershus University Hospital, Lørenskog, Norway; ${ }^{2}$ Acute Clinic, Oslo University Hospital, Oslo, Norway

10.1136/archdischild-2014-307384.347

Background In this study we wanted to evaluate if parents of very-low birth weight (VLBW) infants enrolled in a clinical study developed a lower quality of life compared to parents from a control sample.

Methods We recruited parents of children attending the Norwegian multicenter study for premature nutrition (PreNu). The PreNu study was a randomised clinical nutritional trial, where 50 VLBW-children $(<1500 \mathrm{~g})$ were recruited within the first hours of their life. We also recruited parents of VLBW-children born immediately before and after the recruitment period of the PreNu study, to serve as a control group.

The parents $(n=63)$ were given a questionnaire with validated measures on quality of life (Quality of Life Scale), anxiety and depression (Hospital Anxiety and Depression Scale), fatigue (Lee Fatigue Scale), sleeping disturbance (General Sleep Disturbance Scale), pain (Brief Pain Inventory), comorbidity (SelfAdministered Comorbidity Questionnaire) and hope (Herth Hope Index). The parents were asked to answer as they would have done at the time their children were at the NICU.

Results The response rate was 69\%. T-tests showed no significant difference between the groups on all measures except for quality of life. The PreNu parents rated their quality of life significantly higher than the control group ( $p=0.018$ ).

Conclusion Our fear that the parents of the PreNu-children suffered an intolerable burden seems unfounded. The results suggest that being the parent of a VLBW-child attending a clinical study is not a burden, but may in fact be an enrichment.

\section{PS-051 RANDOMISED CONTROLLED TRIALS IN VERY PRETERM INFANTS: DOES INCLUSION IN THE STUDY RESULT IN ANY LONG-TERM BENEFIT?}

${ }^{1} \mathrm{CM}$ Rüegger, ${ }^{2} \mathrm{~A}$ Kraus, ${ }^{1} \mathrm{~B}$ Koller, ${ }^{3} \mathrm{G}$ Natalucci, ${ }^{3} \mathrm{~B}$ Latal, ${ }^{1} \mathrm{E}$ Waldesbühl, ${ }^{1} \mathrm{JC}$ Fauchère, ${ }^{2} \mathrm{~L}$ Held, ${ }^{1} \mathrm{HU}$ Bucher. 'Division of Neonatology, University Hospital Zurich, Zurich, Switzerland; ${ }^{2}$ Division of Biostatistics, University of Zurich, Zurich, Switzerland; ${ }^{3}$ Child Development Center, University Children's Hospital, Zurich, Switzerland

\subsection{6/archdischild-2014-307384.348}

Background Since the introduction of randomised controlled trials (RCT) in clinical research, there has been discussion of whether enrolled patients have worse or better outcomes than comparable nonparticipants.
Objective To investigate whether very preterm infants randomised to a placebo group in a RCT have equivalent neurodevelopmental outcomes to infants who were eligible but not randomised (eligible NR).

Methods In the course of an RCT investigating the neuroprotective effect of early high dose erythropoietin on the neurodevelopment of very preterm infants, the outcome data of 72 infants randomised to placebo were compared with those of 108 eligible NR infants. Our primary outcome measures were the mental (MDI) and psychomotor (PDI) developmental indices of the Bayley Scales of Infant Development II at 24 months corrected age. The outcomes of the two groups were considered equivalent if the confidence intervals of their mean differences fitted within our \pm 5 point margin of equivalence.

Results Except for a higher socioeconomic status of the trial participants, both groups were balanced for most perinatal variables. The mean difference $(90 \% \mathrm{CI})$ between the placebo and the eligible NR group was -2.1 (-6.1 and 1.9) points for the MDI and -0.8 ( -4.2 and 2.5 ) points for the PDI (in favour of the placebo group). After adjusting for the socioeconomic status, maternal age and child age at follow-up, the mean difference for the MDI was -0.5 (-4.3 and 3.4) points.

Conclusions Our results indicate that the participation of very preterm infants in an RCT is associated with equivalent longterm outcomes compared to non-participating infants.

\section{PS-052 SETTING PRETERM BIRTH RESEARCH PRIORITIES WITH MULTIPLE PROFESSIONS AND SERVICE USERS IN THE UK}

${ }^{1} S$ Uhm, ${ }^{2} \mathrm{~F}$ Alderdice, ${ }^{3} \mathrm{I}$ Brady, ${ }^{1} \mathrm{~B}$ Chambers, ${ }^{4} \mathrm{Z}$ Chivers, ${ }^{5} \mathrm{~S}$ Crowe, ${ }^{6} \mathrm{AL}$ David, ${ }^{7} \mathrm{~S}$ Deshpande, ${ }^{8} \mathrm{C}$ Gale, ${ }^{9} \mathrm{G}$ Gyte, ${ }^{6} \mathrm{CP}$ James, ${ }^{10} \mathrm{~L}$ Duley, ${ }^{11} \mathrm{~J}$ McNeill, ${ }^{12} \mathrm{~A}$ Shennan, ${ }^{13} \mathrm{MA}$ Turner, ${ }^{1} \mathrm{~S}$ Oliver. ${ }^{1}$ Institute of Education, University of London, London, UK; ${ }^{2}$ The Premature Baby Charity for Northern Ireland, TinyLife, Carryduff, UK; ${ }^{3}$ rrish Premature Baby, Irish Premature Baby, Dublin, Ireland; ${ }^{4}$ The Premature and Special Care Baby Charity, Bliss, London, UK; ${ }^{5}$ James Lind Alliance, James Lind Alliance, London, UK; ${ }^{6}$ University College London, Institute for Women's Health, London, UK; ' British Association of Perinatal Medicine, British Association of Perinatal Medicine, London, UK; ${ }^{8}$ Academic Neonatal Medicine, Imperial College, London, UK; ${ }^{9}$ National Childbirth Trust, National Childbirth Trust, London, UK; ${ }^{10}$ University of Nottingham, Nottingham Clinical Trials Unit, Nottingham, UK; ${ }^{11}$ The Premature Baby Charity for Northern Ireland, TinyLife, Carryduf, UK; ${ }^{12}$ Kings College London, Kings College London, London, UK; ${ }^{13}$ Liverpool Women's NHS Foundation Trust, Liverpool Women's NHS Foundation Trust, Liverpool, UK

\subsection{6/archdischild-2014-307384.349}

Background Preterm birth is the most important determinant of adverse infant outcomes. Research agendas in this area have been determined primarily by researchers.

Objectives To identify and prioritise future research areas in preterm birth that are most important to service users and practitioners.

Methods A priority setting partnership was established with families with experience of preterm birth and organisations representing them, obstetricians, neonatologists, midwives and neonatal nurses. Research uncertainties were gathered from surveys and analysis of systematic reviews and clinical guidance. Prioritisation was through voting; final ranking occurred at a facilitated workshop, as advocated by the James Lind Alliance.

Results 593 uncertainties were submitted by 386 respondents (58\% service users, 30\% healthcare professionals and 12\% from those in both roles); 52 were identified from literature 
reviews. After merging similar questions, 104 were distributed for voting. From the 30 most popular uncertainties, the top 15 questions were prioritised in a facilitated workshop These include prevention and prediction of preterm birth, neonatal infection, lung damage, necrotising enterocolitis, preeclampsia, preterm premature rupture of the membranes, optimal neonatal feeding strategy, pain perception and management, a care package at neonatal discharge, emotional and practical support, attachment and bonding, and the best time for cord clamping.

Conclusions These priorities provide guidance to ensure that future research addresses questions that are important to service users and clinicians. Challenges for the priority setting partnership included maximising participation amongst people most affected by preterm birth, the breadth of the topic and securing input from an appropriate range of clinicians.

\section{PS-052a NEURODEVELOPMENTAL OUTCOME OF EXTREMELY PRETERM INFANTS AT 6.5 YEARS OF AGE; EXTREMELY PRETERM INFANTS STUDY IN SWEDEN (EXPRESS)}

${ }^{1} \mathrm{~F}$ Serenius, ${ }^{2} \mathrm{U}$ Ewald, ${ }^{3} \mathrm{~V}$ Fellman, ${ }^{4} \mathrm{M}$ Hafström, ${ }^{5} \mathrm{~K}$ Hellgren, ${ }^{6} \mathrm{~K}$ Källen, ${ }^{7} \mathrm{E}$ Lindberg, ${ }^{8} \mathrm{~K}$ Marsal, ${ }^{9} \mathrm{M}$ Norman, ${ }^{10} \mathrm{E}$ Olhager, ${ }^{11} \mathrm{~K}$ Stjernqvist, ${ }^{12} \mathrm{U}$ Åden, ${ }^{13} \mathrm{~A}$ Farooqi, ${ }^{2} \mathrm{~B}$ Strömberg. 'Women's and Children's Health, Uppsala University, Uppsala, Sweden; ${ }^{2}$ Pediatrics, Uppsala University, Uppsala, Sweden; ${ }^{3}$ Pediatrics, Lund University, Lund, Sweden; ${ }^{4}$ Pediatrics, Sahlgrenska University Hospital, Göteborg, Sweden; ${ }^{5}$ Ophthalmology, Karolinska University Hospital, Stockholm, Sweden; ${ }^{6}$ Centre for Reproductive Epidemiology, Lund University, Lund, Sweden; ${ }^{7}$ Pediatrics, Örebro University, Örebro, Sweden; ${ }^{8}$ Obstetrics Gynecology, Lund University, Lund, Sweden; ${ }^{9}$ Pediatrics, Karolinska University Hospital, Stockholm, Sweden; ${ }^{10}$ Pediatrics, Linköping University, Linköping, Sweden; ${ }^{11}$ Psychology, Lund University, Lund, Sweden; ${ }^{12}$ Pediatrics, Karolinska University Hospital, Stockholm, Sweden; ${ }^{13}$ Pediatrics, Umeå University, Umeå, Sweden

\subsection{6/archdischild-2014-307384.350}

Background Active perinatal care increases the survival of extremely preterm infants but there are concerns that improved survival might increase the rate of disabled survivors.

Objective To determine neurodevelopmental outcome at 6.5 years of age in extremely preterm children (EPT, $<27$ weeks) in a Swedish National cohort.

Design/methods Poulation-based prospective cohort of all EPT children born in Sweden from April 1, 2004, to March 31, 2007. Survivors were assessed and compared with a term-born control group. Of 707 live-born infants, 69\% survived to 6.5 years. Intellectual ability was measured with WISC- IV and results were related to the mean and SD of the controls. Clinical examination and parental questionnaires were used for diagnosis of cerebral palsy, hearing and vision impairments.

Results At a median age of 78 months, 445 of 494 eligible EPT children $(90 \%)$ were assed (59 by chart review). The rates of cerebral palsy, moderate visual impairment, blindness and deafness were $9.2 \%, 5.2 \%, 2.0 \%$ and $0.7 \%$, respectively vs $0.0 \%$, $0.5 \%, 0 \%$ and $0 \%$, respectively among 370 controls. 364 EPT children and 369 controls were formally tested with WISC-IV. Intellectual impairment $<-2$ SD but $>-3 \mathrm{SD}$, and $<-3 \mathrm{SD}$ was $9 \%$ and $19 \%$, respectively vs $1.9 \%$ and $0 \%$, respectively among controls. In 445 EPT children either formally assessed or by chart review, the rates of moderate and severe neurodevelopmental disabilities were $19 \%$ and $11 \%$, respectively compared with $2.4 \%$ and $0 \%$, respectively among control children.

Conclusion Disability rates are comparable to similar studies that report lower survival rates.

\section{Feeding Regimen}

\section{PS-053 VARIABILITY IN ADIPOKINES PROFILE OF NEWBORNS AND THEIR MOTHERS AFTER DHA SUPPLEMENTATION IN PREGNANCY}

${ }^{1}$ E Martín Alvarez, ${ }^{1} \mathrm{M}$ Peña-Caballero, ${ }^{1} \mathrm{JA}$ Hurtado-Suazo, ${ }^{2} \mathrm{~N}$ Kajarabille, ${ }^{3} \mathrm{~F}$ Lara-Villoslada, ${ }^{2} \mathrm{JJ}$ Ochoa. ${ }^{1}$ Paediatric, Hospital Universitario Virgen de Las Nieves, Granada, Spain; ${ }^{2}$ Nutrition, Institute of Nutrition and Food Technology "José Mataix", Granada, Spain, ${ }^{3}$ R\&D Department, Lactalis Puleva, Granada, Spain

\subsection{6/archdischild-2014-307384.351}

Background/aims Most studies of DHA supplementation during pregnancy and infant development are focused on visual and neural development. However, scarce information is available about the influence of DHA supplementation on adipokines expression, which are related to adipose tissue metabolism and obesity.

Aim Evaluate the effect of DHA supplemented dairy drink consumption during pregnancy and breastfeeding on the expression of several adipokines in mothers (pregnancy, delivery and breastfeeding) and their newborns (birth and 2.5 months of age).

Methods 60 women were randomly assigned to two intervention groups: A) Control Group ( $\mathrm{n}=30)$; B) Supplemented Group ( $\mathrm{n}=30)$ : The women took 2 glasses/day of the supplemented drink (400 mg DHA/day). Dietary intervention began in week 28th of pregnancy and concluded when breastfeeding stopped. Samples of blood were obtained from: the mothers, the umbilical vein and artery; the newborn at 2.5 months postpartum. Adiponectin, resistin, leptin and active PAI-1 plasma levels were determined using a panel from Luminex xMAP technology. Results Adiponectin was higher in the supplemented group in umbilical cord artery, whereas active-PAI showed a lower value in this group, although we observed an increase in mother's blood during delivery. Resistin did not show any difference. Leptin was higher in the supplemented group in umbilical cord.

Conclusion The most noteworthy result is the effect of DHA supplementation in umbilical cord artery adipokine levels, increasing the adiponectin and decreasing the active PAI-1. In addition, active PAI-1 increases in mothers during delivery. It is also interesting the increase in the supplemented group of leptin.

\section{\begin{tabular}{|l|l}
\hline PS-054 & VSL\#3 SUPPLEMENTATION TO MOTHERS DURING
\end{tabular} PREGNANCY AND BREAST FEEDING IMPROVES COLICS AND REGURGITATION IN NEWBORNS, PERHAPS BY TGF-B MODULATION}

${ }^{1} \mathrm{ME}$ Baldassarre, ${ }^{2} \mathrm{P}$ Mastromarino, ${ }^{3} \mathrm{~A}$ Miccheli, ${ }^{4} \mathrm{M}$ Fanelli, ${ }^{1} \mathrm{~A}$ Dileone, ${ }^{1} \mathrm{P}$ Drimaco, ${ }^{1} \mathrm{~N}$ Laforgia. 'Department of "Scienze Biomediche ed Oncologia Umana"- Neonatology and NICU Section, University of Bari, Bari, Italy; ${ }^{2}$ Department of Public Health and Infectious Diseases, University "La Sapienza", Rome, Italy; ${ }^{3}$ Department of Chemistry, University "La Sapienza", Rome, Italy; ${ }^{4}$ Department of Internal Medicine and Public Health-Section of Nuclear Medicine-Medical Statistics, University of Bari, Bari, Italy

\subsection{6/archdischild-2014-307384.352}

Aim To evaluate the influence of maternal supplementation with VSL\#3 on digestive events in newborns and cytokines levels in colostrum and mature breast milk.

Methods This pilot double-blind, randomised, placebo-controlled clinical trial (clinicaltrials.gov: NCT01367470) enrolled four weeks before expected delivery healthy pregnant women that received daily oral VSL\#3 (PTM) or placebo (CM) supplementation until four weeks after delivery. In milk samples we 